\title{
FISIOTERAPIA NA RESIDÊNCIA MULTIPROFISSIONAL EM SAÚDE DA FAMÍLIA: RELATO DE EXPERIÊNCIA
}

PHYSICAL THERAPY IN THE FAMILY HEALTH MULTIPROFESSIONAL RESIDENCY PROGRAM: EXPERIENCE REPORT

FISIOTERAPIA EN EL PROGRAMA DE RESIDENCIA MULTIPROFESIONAL EN SALUD DE LA FAMILIA: INFORME DE EXPERIENCIA

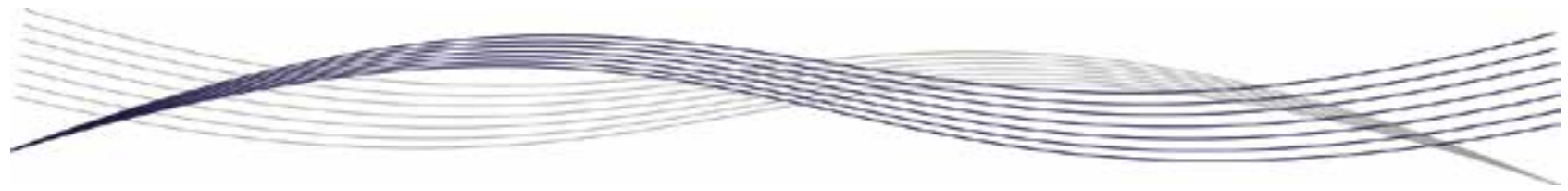

Palavras-chave:

Residência; Atenção Básica; Fisioterapia.

Keywords: Residency; Primary Health Care; Physical Therapy.

Palabras clave: Residencia; Atención Primaria de Salud; Fisioterapia.

Submetido:

$27 / 03 / 2018$

Aprovado:

30/09/2018

Autor(a) para

Correspondência:

Ana Mayara Barros Oliveira

R. Teresina, 58

Nova Parnaíba

Parnaíba (PI)

CEP: 64218-680

E-mail:

anamayara7@hotmail.com
RESUMO

Os programas de residência multiprofissional em saúde estimulam competências e habilidades para ações de atenção, assistência e gestão em saúde. Este artigo descreve a experiência de uma fisioterapeuta residente em uma equipe multiprofissional da Estratégia Saúde da Família (ESF). Trata-se de relato de experiência, com caráter retrospectivo $e$ descritivo, realizado no biênio 2016-2017. Descrevem-se planejamentos e ações multidisciplinares junto às equipes de residentes e de profissionais do serviço de saúde nos três territórios contemplados, além de ações específicas de protocolos fisioterapêuticos na atenção básica, nos diversos ciclos da vida, voltadas à tríade ensino/serviço/comunidade. Concluise que a fisioterapia se inclui na atenção básica à saúde com atuação específica e em equipe, assumindo papel preventivo - de reabilitação $e$ promoção da saúde. Estudos interprofissionais, em diferentes realidades socioeconômicas e níveis de saúde, tendem a contribuir com a formulação de protocolos para ampliar as ações fisioterapêuticas em saúde.

\footnotetext{
1. Fisioterapeuta. Programa de Residência Multiprofissional em Atenção Básica/Saúde da Família da Universidade Federal do Piauí (UFPI). Parnaíba (PI), Brasil.

2. Fisioterapeuta. Professora da UFPI. Parnaíba (PI), Brasil
} 


\section{ABSTRACT}

Health care multiprofessional residency programs stimulate skills and abilities for health care, assistance, and management actions. This article describes the experience of a physical therapist as a resident in a multiprofessional team of the Family Health Strategy (FHS). This is an experience report, with a retrospective and descriptive nature, conducted in the biennium 2016-2017. Multidisciplinary plans and actions along with the teams of residents and practitioners at the health service in the three territories concerned are described, as well as specific actions of physical therapy protocols in primary health care, in the various life cycles, aimed at the triad teaching/service/ community. It is concluded that physical therapy is included in primary health care by means of specific and teambased work, playing a preventive role - aimed at health rehabilitation and promotion. Interprofessional studies, at various socioeconomic contexts and health care levels, tend to contribute to devising protocols in order to expand physical therapy actions in health care.

\section{RESUMEN}

Los programas de residencia multiprofesional en salud estimulan competencias y habilidades para acciones de cuidado, asistencia y gestión. Este artículo describe la experiencia de una fisioterapeuta residente en un equipo multiprofesional de la Estrategia Salud de la Familia (ESF). Este es un informe de experiencia, con carácter retrospectivo y descriptivo, realizado en el bienio 2016-2017. Se describen planes y acciones multidisciplinarios junto con los equipos de residentes y de profesionales del servicio de salud en los tres territorios en cuestión, así como las acciones específicas de protocolos fisioterapéuticos en la atención primaria de salud, en los diversos ciclos de la vida, dirigidas a la tríada enseñanza/servicio/comunidad. Se concluye que la fisioterapia se incluye en la atención primaria de salud mediante trabajo específico y en equipo, jugando un papel preventivo - dirigido a la rehabilitación y promoción de la salud. Estudios interprofesionales, en diferentes realidades socioeconómicas y niveles de atención de salud, tienden a contribuir a la formulación de protocolos para expandir las acciones fisioterapéuticas en salud.

\section{INTRODUÇÃO}

Os programas de residência multiprofissional em saúde, desde sua criação pelo Ministério da Saúde, em 2002, favorecem a formação qualificada de jovens profissionais da saúde, não médicos, para atuação no Sistema Único de Saúde (SUS) ${ }^{1}$. Tratase de uma modalidade de pós-graduação lato sensu que tem por finalidade a formação coletiva em serviço, mediante acompanhamento e supervisão, com vistas a qualificar profissionais comprometidos com os princípios e as diretrizes de integralidade do cuidado no âmbito do SUS ${ }^{2-3}$.

Os programas de residência multiprofissional em saúde proporcionam aos residentes habilidades e especialidades específicas, estimulando competências para ações de atenção, assistência e gestão em saúde $e^{4}$ Seu caráter multidisciplinar possibilita uma relação recíproca entre as diversas intervenções técnicas e a interação entre profissionais de diferentes áreas, facilitando a resolução de problemas à medida que agrupa vários saberes com um mesmo objetivo. Isso proporciona um olhar minucioso e promove a aplicação dos conhecimentos específicos necessários para a resolução dos problemas de saúde da população ${ }^{5}$.

A abrangência e ampliação do escopo das ações desenvolvidas na atenção básica à saúde decorrem das atividades desenvolvidas pelas equipes da Estratégia Saúde da Família (ESF), apoiadas por equipes multiprofissionais do Núcleo Ampliado de Saúde da Família e Atenção Básica $\left(\right.$ Nasf-AB) ${ }^{6}$ no qual o fisioterapeuta está inserido ${ }^{7}$.

Especificamente em relação a esse aspecto, a inclusão do fisioterapeuta em programas de atenção básica na rede pública de saúde se mostra relevante, em virtude das contribuições que esse profissional pode trazer à população. Com formação clínica generalista e capacidade de atuar em todos os níveis de atenção à saúde, ele não se restringe a ações curativas ou reabilitadoras, podendo atuar em programas de prevenção de doenças ou promoção da saúde ${ }^{8}$.

Assim, este artigo descreve a experiência de uma fisioterapeuta residente em uma equipe multiprofissional da ESF, com atividades realizadas no biênio 2016-2017. 


\section{METODOLOGIA}

Este estudo constitui um relato de experiência, de caráter retrospectivo e descritivo, com atividades realizadas no biênio 2016-2017.

0 Programa de Residência Multiprofissional em Atenção Básica/Saúde da Família (PRMAB/SF) da Universidade Federal do Piauí (UFPI) teve início em março de 2016, no Campus Ministro Reis Velloso, em Parnaíba-PI, contemplando as seguintes categorias profissionais: Enfermagem; Farmácia; Fisioterapia; e Psicologia. Sua primeira turma foi composta por 12 residentes, distribuídos em 3 equipes com 1 representante de cada área.

0 PRMAB/SF decorreu da integração ensino/ serviço/comunidade, viabilizada pela parceria entre a UFPI e a Secretaria Municipal de Saúde (SMS) de Parnaíba. As equipes de residentes têm 40 horas em serviço e 20 horas de atividades teóricas, cumprindo uma carga horária de 60 horas semanais - a carga horária total do programa é de 5.760 horas (24 meses).

A cidade de Parnaíba se localiza no litoral do Piauí e tem uma população estimada em 145.705 habitantes ${ }^{9}$. 0 território se divide em 4 distritos de saúde, compostos por 41 equipes da ESF, distribuídas em 35 unidades básicas de saúde (UBS), e 4 equipes do Nasf-AB ${ }^{10}$ - todas com fisioterapeuta (7 no total). Segundo dados obtidos junto ao Conselho Regional de Fisioterapia e Terapia 0cupacional do Piauí (Crefito 14), até 2017, o Piauí tinha 2.878 fisioterapeutas (232 em Parnaíba) ${ }^{11}$.

Operacionalmente, cada equipe de residentes foi lotada em 3 UBS distintas, indicadas pela SMS, de modo que os residentes passaram a fazer parte das equipes de saúde, interagindo com os demais profissionais das equipes da ESF e do Nasf-AB.

Inicialmente, houve acolhimento dos residentes por parte dos professores, tutores e preceptores;

\footnotetext{
A territorialização possibilita que se conheça a história da comunidade...
}

familiarização com a proposta da residência e os profissionais da rede de atenção básica do município; e visita às UBS de referência de alguns dos territórios, para que as equipes de residentes conhecessem as áreas de atuação. Em seguida, teve início o processo de territorialização nas áreas abrangidas pela UBS Tabuleiro (Módulo 25 - Bairro Tabuleiro), UBS João XXIII (Módulo 18 - Bairro João XXIII) e UBS Motorista Francisco Diassis (Módulo 33 - Bairro Planalto), para elaborar o plano de intervenção a partir da identificação das necessidades da população atendida.

\section{RESULTADOS E DISCUSSÃO}

A territorialização possibilita que se conheça a história da comunidade, compreendendo suas crenças, costumes e valores e fortalecendo, assim, a cultura popular local a partir dos atores sociais que protagonizam essa ação. Isso também abre a possibilidade de monitoramento das ações da equipe de saúde e a busca de parcerias intersetoriais e com outros segmentos da sociedade, o que favorece o controle das ações no território. A ESF deve conhecer os condicionantes de saúde da área, bem como identificar os riscos e as vulnerabilidades de grupos, famílias e pessoas ${ }^{12}$.

Para a territorialização, acompanhamos de perto - trabalho dos agentes comunitários de saúde (ACS), um importante elo entre a comunidade e o serviço de saúde. Com o auxílio dos ACS, conhecemos os principais dispositivos sociais dos bairros e realizamos visitas domiciliares e rodas de quarteirão em locais aleatórios da área, com o intuito de colher informações sobre a comunidade, levantando suas dificuldades e potencialidades para planejar as ações no do território.

A comunidade pôde tirar dúvidas e expressar sua opinião, possibilitando que os membros da equipe de residentes compreendessem a realidade social e o processo saúde-doença a partir do olhar e dos sentimentos dos moradores. 0 modo como as pessoas convivem no território além de seu acesso ao serviço de saúde, seus problemas mais prevalentes e o que esperam de melhorias em termos de saúde e para o bairro como um todo desperta um olhar com empatia diante das informações para que as ações planejadas possam suprir as necessidades identificadas.

A territorialização possibilitou caracterizar a população e seus problemas de saúde, construir o diagnóstico situacional dos territórios e pôr em 
prática os planos de intervenção de acordo com as necessidades encontradas, assim como fortalecer o vínculo entre a equipe de saúde e a população adscrita. 0s encontros proporcionaram estreitamento das relações e maior interação entre os sujeitos. 0 fortalecimento do vínculo entre profissionais e usuários trouxe maior confiança, favorecendo a produção do cuidado e a melhora da qualidade da atenção.

Para a efetividade das ações em saúde e o direcionamento das atividades, mostra-se importante identificar os determinantes sociais que influenciam os fatores de adoecimento da população. Os determinantes sociais em saúde correspondem a situações de vida e trabalho dos indivíduos e grupos relacionados com sua condição de saúde, considerando os aspectos sociais, econômicos, culturais étnicos/raciais, psicológicos e comportamentais que influenciaram a ocorrência de problemas de saúde e seus fatores de risco na população $0^{13}$. Essa identificação foi possível por meio do processo de territorialização e do conhecimento das demandas que chegam à UBS.

$\mathrm{Na}$ realidade vivenciada, as equipes da ESF eram formadas por médico, enfermeiro, auxiliar e técnico de enfermagem, odontólogo, auxiliar de consultório dentário, técnico em higiene bucal, atendente social e ACS, sendo que as outras categorias profissionais contempladas pelo PRMAB/SF, como fisioterapeuta e psicólogo, estavam inseridas apenas na equipe do Nasf-AB e o farmacêutico não estava incluído nessas equipes.

Observou-se em todas as UBS que as equipes de saúde priorizavam as ações curativas e atendimento das demandas do dia, não valorizando as atividades de prevenção de doenças e promoção da saúde, alegando falta de tempo e excesso de trabalho. Mesmo sabendo da importância da realização dessas ações, os profissionais mostravam dificuldades para propor soluções que as viabilizassem.

Dentre os profissionais, apenas os ACS participavam das atividades desenvolvidas pelas residentes. Assim, tornou-se um desafio compartilhar nossos conhecimentos e experiências, trazer novas perspectivas de trabalho, diferentes daquelas do cotidiano das equipes, e convencer os profissionais da saúde a abraçar nossas propostas. A inclusão dos residentes nas equipes da ESF ampliou o escopo dos serviços ofertados, fortalecendo as ações com ênfase na promoção da saúde e prevenção de doenças, e sua maior disponibilidade nas UBS

\section{...tornou- \\ se um desafio compartilhar nossos conhecimentos $e$ experiências...}

favorece o acompanhamento e a solução dos casos atendidos.

Para efetivar o cuidado integral em saúde e a interdisciplinaridade das ações, garantindo a interação entre vários conhecimentos técnicos e específicos, contar com uma equipe multiprofissional é fundamental. A cooperação entre diversos indivíduos detentores de diferentes saberes abre espaço para soluções ou propostas de intervenção, as quais não poderiam ser produzidas por qualquer profissional de modo isolado. A multiprofissionalidade surge como uma estratégia de reorganização dos serviços de saúde, focada na prática integrada entre diversos profissionais, visando a um atendimento integral capaz de sintonizar toda a complexidade envolvida no processo de cuidado à saúde ${ }^{14}$.

0 fisioterapeuta encontra, ainda, certa dificuldade de inserção nas equipes da ESF devido a seu histórico de profissional reabilitador, cuja formação tem pouca ênfase em saúde da família. Sua participação na equipe é relevante para 0 esclarecimento da profissão não apenas em termos de sua função reparadora, com foco na assistência individual, mas também quanto à sua função resolutiva na saúde funcional, por meio de ações preventivas $^{15}$.

A inclusão do fisioterapeuta nas equipes da ESF via residência possibilita a desmistificação de seu estigma de tratador/reabilitador diante dos demais profissionais da saúde e dos usuários dos serviços. A experiência de trabalho em equipe multiprofissional, com intercâmbio de experiências interprofissionais e entre profissionais da saúde e sujeitos da comunidade proporciona um olhar diferente no cuidado à saúde, deixando de enfocar o atendimento individual e enxergando as potencialidades do atendimento coletivo.

As ações específicas da fisioterapia foram desenvolvidas por meio das atividades em grupo, visitas domiciliares, atendimento compartilhado e atendimento fisioterápico individualizado - quando necessário. 
A proposição e formação de grupos de saúde era discutida com a equipe de acordo com as necessidades trazidas e as demandas locais. 0s ACS ficavam responsáveis por divulgar e convidar a comunidade, porém, no início havia dificuldades em relação à participação e aderência do públicoalvo, seja por falta de interesse, disponibilidade, horário, distância ou outros motivos. Várias foram as propostas de grupos de saúde. Alguns tiveram êxito e tornaram-se fixos, outros não tiveram continuidade por falta de adesão, mas mesmo assim insistíamos nas atividades, sempre adaptando e inovando as práticas de saúde para despertar o interesse da comunidade em participar dos encontros.

Em um dos bairros assistidos, uma das principais queixas da população era a distância da UBS de seu domicílio, o que dificultava o acesso e limitava a procura dos serviços a casos extremos. Nossa proposta foi levar os residentes até as comunidades, com a ajuda dos ACS, em dispositivos sociais bem localizados, como igrejas, ginásios, escolas ou as casas de moradores para atividades como medição de pressão arterial sistêmica e glicemia capilar, além de rodas de conversa para orientações de cuidado à saúde.

Primeiramente, destacam-se os grupos de gestantes, com encontros no dia da consulta de pré-natal. 0 foco das orientações fisioterápicas recaía sobre postura corporal, principais alterações corporais durante a gestação, preparo para o parto, cuidados nos períodos pré e pós-parto, exercícios para o assoalho pélvico, importância da prática de exercício físico a partir do segundo trimestre de gestação, quando não há contraindicações, alongamentos e exercícios de fortalecimento para evitar dores articulares, cãibras e edemas. Ao final das orientações eram realizados exercícios respiratórios, alongamentos e exercícios de relaxamento e fortalecimento ${ }^{16}$.

Em segundo lugar, os grupos de idosos tinham o propósito de despertar o interesse dos usuários

\section{Os temas das atividades eram definidos de acordo com a necessidade da população local...}

no autocuidado, por meio de medidas educativas e atividades dinâmicas de interação. Mesmo sendo a proposta inicial a formação de grupos terapêuticos voltados aos idosos, também houve participação de adultos jovens, com predomínio do sexo feminino.

Antes das atividades ocorria a medição da pressão arterial sistêmica e uma roda de conversa, conduzida cada dia por uma das profissionais da saúde, com algum tema pertinente sobre cuidado à saúde. As atividades práticas ficavam sob a responsabilidade da fisioterapeuta residente.

Essas atividades consistiam em jogos, danças, circuitos funcionais, exercícios para memória e concentração, alongamentos e práticas corporais com auxílio de recursos simples e de fácil aquisição, como bolas, bastões, balões, bambolês e garrafas PET, entre outros.

As práticas propostas buscavam trabalhar os aspectos físicos, motores, mentais, sociais e afetivos, valorizando o desenvolvimento integral de habilidades e capacidades e estimulando a adoção de hábitos de vida saudável e um estilo de vida fisicamente ativo. Os grupos tinham o objetivo de promover a construção do autocuidado, gerar autonomia e estabelecer vínculos com a comunidade, pautados em troca de saberes, monitoramento e acompanhamento das condições clínicas, com consequente melhora da qualidade de vida ${ }^{17}$.

Além das atividades coletivas, também houve escuta e acolhimento individual desses usuários, identificando as demandas e realizando os devidos encaminhamentos, além do convite para que a população frequentasse a UBS e conhecesse os serviços disponíveis.

0 s temas das atividades eram definidos de acordo com a necessidade da população local, abordando os diferentes ciclos da vida.

Em termos de saúde da criança, o foco recaiu sobre cuidado com a higiene íntima, prevenção e tratamento de verminoses e parasitoses e orientações para evitar e tratar doenças respiratórias.

As ações voltadas à saúde da mulher enfocaram a importância da realização de exames preventivos, cuidado com a saúde íntima, prevenção de infecções sexualmente transmissiveis (IST), planejamento familiar, orientações sobre disfunções sexuais e alterações de funções, como incontinência urinária.

$\mathrm{Na}$ saúde do homem, a atenção se voltou a prevenção de IST, prevenção do câncer de próstata, promoção da saúde e importância da realização de consultas periódicas, visto que esse público costuma 
cuidar menos de sua saúde e visitar as UBS com menos frequência.

Foram realizadas ações do HiperDia, que instrumentaliza a prática de atendimento aos usuários hipertensos e/ou diabéticos e gera informações de acordo com faixa etária, sexo, medicamentos utilizados e acompanhamento da pressão arterial sistêmica ${ }^{18-19}$, com orientações sobre cuidados com alimentação, importância da prática de exercício físico, cuidado com o pé diabético, uso racional de medicação e importância do monitoramento da pressão arterial sistêmica e glicemia.

$\mathrm{Na}$ saúde do idoso, as ações foram voltadas a orientações sobre prevenção de quedas e incapacidades, cuidados com articulações, alterações do envelhecimento, atividades lúdicas de concentração e memória.

No início, as atividades tinham o formato de palestra educativa, porém, constatou-se a necessidade de utilizar recursos como música, confecção de cartazes, exposição de objetos, jogos e práticas corporais para adotar atividades mais dinâmicas que despertassem o interesse e a interação dos usuários. As rodas de conversa dentro dos territórios constituíam uma oportunidades para o fortalecimento de vínculo entre a equipe e a comunidade, pois havia maior aproximação, com contato mais próximo e a quebra do padrão de atendimento individual dentro do consultório.

No ambiente das UBS ocorriam abordagens nas salas de espera com os usuários que estavam aguardando consulta médica ou de enfermagem. As dinâmicas e os temas eram definidos de acordo com a demanda de atendimentos do dia e articulados previamente com a equipe da ESF durante as reuniões de planejamento mensal.

0 acolhimento nas salas de espera tem o objetivo de garantir um cuidado humanizado, proporcionando aproximação cada vez maior entre a comunidade e os serviços de saúde. 0 s profissionais têm a oportunidade de adotar ações que extrapolam o cuidado, proporcionando melhora da qualidade de atendimento, garantindo melhor acolhimento aos usuários, estabelecendo vínculos e amenizando o desgaste físico e emocional associado ao tempo de espera por qualquer atendimento 20 . Deve-se ter um espaço adequado para compartilhamento e troca de experiências entre profissionais da saúde e usuários. Os profissionais da saúde assumem a função de facilitadores do processo em vez da postura de detentores do saber.
As visitas domiciliares sempre ocorriam com mais de um residente, além das atividades coletivas e individuais anteriormente descritas, para que houvesse uma visão ampliada acerca da condição de saúde do usuário e da família, não se limitando ao motivo que levou o ACS a solicitar a visita. A maioria das visitas identificou demandas em mais de um membro da família, o que possibilitou uma atenção integral para cada sujeito da comunidade e o fortalecimento de vínculo com toda a família, além de favorecer a compreensão de aspectos importantes da dinâmica das relações familiares.

A visita domiciliar proporciona ao profissional a oportunidade de conhecer o espaço da família e identificar suas necessidades e potencialidades. Busca ampliar a visão das condições reais de vida da família, a interação entre os familiares, os costumes e as crenças, o que torna a experiência enriquecedora para todos os envolvidos ${ }^{21}$.

Nas visitas domiciliares, as ações da fisioterapeuta residente envolviam pessoas com restrição de mobilidade ou acamadas e orientações para seus cuidadores domiciliares, solicitação de cadeiras de rodas, cadeiras de banho e/ou órteses para auxílio de marcha. Quando necessário, o atendimento domiciliar tinha acompanhamento semanal ou diário (fisioterapia respiratória e/ou motora). Quando se constatou a necessidade de acompanhamento por serviço especializado, o encaminhamento era solicitado ao médico após discussão do caso clínico junto à equipe da UBS.

Nos atendimentos fisioterapêuticos realizados na UBS houve predomínio de crianças hipersecretivas, encaminhadas por profissionais da própria equipe após visitas domiciliares.

As manobras e intervenções cinesioterápicas, realizadas no ambiente da UBS, envolveram a aplicação de técnicas convencionais (drenagem postural, vibração manual ou mecânica, percussão e padrões ventilatórios), a fim de remover secreção brônquica, otimizar a ventilação pulmonar e melhorar o padrão respiratório da criança, também podendo

$$
\begin{gathered}
\text { Deve-se ter } \\
\text { um espaço } \\
\text { adequado para } \\
\text { compartilhamento } \\
\text { e troca de } \\
\text { experiências... }
\end{gathered}
$$


utilizar recursos lúdicos, como bola de sabão, apito, língua de sogra, para facilitar a aceitação do tratamento 22 . Os casos mais complexos eram encaminhados a serviço especializado de média ou alta complexidade.

Junto à enfermagem foram realizadas consultas compartilhadas de puericultura, auxiliando a avaliação do desenvolvimento neuropsicomotor das crianças. No HiperDia, buscou-se contribuir com avaliação e orientações, como prática de exercício físico, alimentação, monitoramento da glicemia e da pressão arterial sistêmica e cuidado com o pé diabético. Em muitos atendimentos foram identificados casos de pacientes com uso irracional de medicação, sendo solicitado acompanhamento da residente farmacêutica para as orientações medicamentosas adequadas.

Outras vivências, em que as consultas compartilhadas proporcionaram um olhar ampliado ao estado de saúde do usuário e maior efetividade nos casos acompanhados, ocorreram nas consultas de prevenção do câncer de colo uterino, para esclarecer as mulheres acerca dos músculos do assoalho pélvico e detectar casos de disfunção dessa musculatura. Durante as avaliações foram identificados muitos casos de incontinência urinária e prolapso uterino, sendo adotadas as devidas orientações, além da prescrição de exercícios para fortalecimento dos músculos do assoalho pélvico e da solicitação de retorno para acompanhamento da efetividade do tratamento ${ }^{23}$.

A incontinência urinária constitui um problema de saúde pública com grande incidência entre as mulheres, sendo pouco relatado pelas usuárias devido ao constrangimento durante a consulta e a falta de questionamento por parte dos profissionais da saúde ${ }^{24}$, sendo observado durante os atendimentos. A assistência fisioterapêtica por meio de uma abordagem educacional, que inclui informações sobre - funcionamento e fortalecimento dos músculos do assoalho pélvico e a prescrição de exercícios específicos, mostra-se eficaz como tratamento para pacientes com disfunção uroginecológica, visto que durante muito tempo as ações cirúrgicas e medicamentosas foram a base do tratamento da incontinência urinária ${ }^{23}$.

0 trabalho, junto às equipes multiprofissionais de atenção básica à saúde, proporcionou a vivência de um espaço de troca de saberes, compartilhamento de conhecimentos e experiências únicas. Esse tipo de vivência não faz parte da formação em nível de

\section{...as consultas \\ compartilhadas \\ proporcionaram um \\ olhar ampliado...}

graduação e facilita a compreensão de que o cuidado à saúde transcende a consulta no consultório, exigindo dos profissionais da saúde, além do conhecimento técnico inerente à sua área de atuação, a capacidade de lidar com as diferenças e o comprometimento com a adoção de novas práticas e processos no setor saúde.

A integração ensino/serviço/comunidade na formação de recursos humanos em saúde prevê a capacitação de profissionais aptos para o trabalho em equipe, com ênfase na integralidade do cuidado. A obtenção de experiência dentro dos serviços se mostra tão importante quanto a formação acadêmica dos profissionais da saúde ${ }^{25}$.

A oferta de novos serviços, proporcionada pela inserção dos residentes nas UBS: a) permitiu que a comunidade desfrutasse de atendimentos especializados com maior facilidade; b) promoveu um contato mais próximo com os profissionais das equipes; e c) levou a maior participação dos usuários nas ações de prevenção de doenças e promoção da saúde.

Vale registrar que a adesão às ações de promoção da saúde por parte da comunidade e dos próprios profissionais da saúde foi crescente, na medida em que se observaram mudanças favoráveis e efetivas tanto em sua saúde física quanto mental, por meio de aprendizagem e adoção de novos hábitos e condutas no cuidado à saúde, favorecendo a conscientização pessoal acerca do processo de produção da saúde e da qualidade de vida.

0 trabalho junto às equipes da ESF trouxe, além da experiência de trabalho em equipe, a oportunidade de conhecer melhor as inúmeras possibilidades de atuação do fisioterapeuta e de apresentá-las aos usuários dos serviços e aos demais profissionais da saúde. Destaca-se a importância do interesse dos usuários nas atividades propostas, da evolução dos casos individuais e da melhora dos aspectos clínicos dos grupos acompanhados, além do aumento da procura pelos serviços de fisioterapia dentro das 
UBS, do encaminhamento de outros profissionais e do reconhecimento de todos acerca da importância da inclusão do fisioterapeuta nas equipes da ESF.

\section{CONCLUSÃO}

A vivência descrita neste artigo indica que a atuação do fisioterapeuta na atenção básica com ênfase em saúde da família e comunidade contempla a tríade ensino/serviço/comunidade em todos os ciclos da vida, com ações interprofissionais em distintos territórios com as mais diversas demandas de atenção e cuidado à saúde, tanto por meio de trabalho em equipe quanto de procedimentos específicos de protocolos fisioterapêuticos.

A continuidade de atividades exitosas e a operacionalização de serviços pautados nos princípios de referência e contrarreferência demandam fortalecimento por parte tanto dos profissionais da saúde como da própria comunidade.

0 registro de experiências como essa corrobora os princípios e as diretrizes de integralidade do cuidado no âmbito do SUS, sob a perspectiva da promoção da saúde, da prevenção de doenças e agravos e da cura e reabilitação em nível primário, ajudando os profissionais da saúde envolvidos a ampliar seu olhar acerca das diversas formas de atuação.

As possibilidades identificadas nos programas de residência multiprofissional em saúde subsidiam o desenvolvimento de estudos multiprofissionais, realizados em diversas realidades socioeconômicas e nos vários níveis de atenção à saúde, contribuindo com a formulação de protocolos para ampliar as ações especializadas em saúde.

\section{CONTRIBUIÇÃO DAS AUTORAS}

Ana Mayara Barros Oliveira contribuiu com a realização da pesquisa, o delineamento do estudo e a redação do manuscrito. Natasha Teixeira Medeiros contribuiu com o delineamento do estudo e a redação e revisão crítica do manuscrito.

\section{REFERÊNCIAS}

1. Costa ML, Silva RF, Lima VV, Ogata MN. Residência multiprofissional em saúde e o mundo do trabalho do fisioterapeuta. Tempus (Brasília) [serial on the internet]. 2016 [cited 2018 Nov 5];10(4):101-10. Available from: file:///D:/2025-6858-1-PB.pdf

2. Martins GDM, Caregnato RCA, Barroso VLM, Ribas DCP. Implementação de residência multiprofissional em saúde de uma universidade federal: trajetória histórica. Rev Gaúch Enferm [serial on the internet]. 2016 [cited 2018 Nov 5];37(3):1-8. Available from: http://www.scielo.br/pdf/rgenf/v37n3/0102-6933rgenf-1983-144720160357046.pdf

3. Casanova IA, Batista NA, Ruiz-Moreno L. Formação para o trabalho em equipe na residência multiprofissional em saúde. ABCS Health Sci [serial on the internet]. 2015 [cited 2018 Nov 5];40(3):22933. Available from: https://www.portalnepas.org. br/abcshs/article/viewFile/800/695

4. Soares RSA, Penna MA, Pinno C, Durgante VL, Saul AMR, Farão EMD, et al. Vivências de residentes enfermeiros no programa de residência multiprofissional em saúde. Saúde (Santa Maria) [serial on the internet]. 2017 [cited 2018 Nov 5];43(1):13-21. Available from: https://periodicos. ufsm.br/revistasaude/article/view/14826/pdf

5. Castro SS, Cipriano Junior G, Martinho, A. Fisioterapia no Programa de Saúde da Família: uma revisão e discussões sobre a inclusão. Fisioter Mov [serial on the internet]. 2006 [cited 2018 Nov 5];19(4):55-62. Available from: file:///D:/1879438351-1-PB.pdf

6. Brasil. Portaria GM n. 2.436, de 21 de setembro de 2017. Aprova a Política Nacional de Atenção Básica, estabelecendo a revisão de diretrizes para a organização da Atenção Básica, no âmbito do Sistema Único de Saúde (SUS). Diário Oficial da União, Brasília (2017 Sep 21); Sec 1.

7. Barbosa EG, Ferreira DLS, Furbino SAR, Ribeiro EEN. Experiência da fisioterapia no Núcleo de Apoio à Saúde da Família em Governador Valadares, MG. Fisioter Mov [serial on the internet]. 2010 [cited 2018 Nov 5];23(2):323-30. Available from: http:// www.scielo.br/pdf/fm/v23n2/15.pdf

8. Maia FES, Moura ELR, Madeiros EC, Carvalho RRP, Silva SAL, Santos GR. A importância da inclusão do profissional fisioterapeuta na atenção básica de saúde. Rev Fac Ciênc Méd Sorocaba [serial on the internet]. 2015 [cited 2018 Nov 5];17(3):110-5. Available from: http://revistas.pucsp.br/index.php/ RFCMS/article/view/16292/pdf

9. Brasil. Censo 2010. Rio de Janeiro: Instituto Brasileiro de Geografia e Estatística; 2010.

10. Brasil. Cadastro Nacional de Estabelecimentos de Saúde - CNES. Brasília (DF): Ministério da Saúde; 2018.

11. Brasil. Crefito 14. Teresina: Conselho Regional de Fisioterapia e Terapia 0cupacional do Piauí; 2017. 
12. Ramos MJM, Bezerra MIC, Paiva GM. Saúde, ambiente e qualidade de vida: reflexões da experiência da residência multiprofissional em saúde da família e comunidade de Fortaleza-CE. Cadernos ESP [serial on the internet]. 2013 [cited 2018 Nov 5];7(2):53-65. Available from: http://www.esp. ce.gov.br/cadernosesp/index.php/cadernosesp/ article/view/115/84

13. Bosi MLM, Melo AKS, Carvalho LB, Ximenes VM, Godoy MGC. Determinantes sociais em saúde (mental): analisando uma experiência não governamental sob a ótica de atores implicados. Rev Bras Epidemiol [serial on the internet]. 2014 [cited 2018 Nov 5];(Suppl DSS):126-35. Available from: http://www. scielo.br/pdf/rbepid/v17s2/pt 1415-790X-rbepid17-s2-00126.pdf

14. Salvador AS, Medeiros CS, Cavalcanti PB, Carvalho RN. Construindo a multiprofissionalidade: um olhar sobre a residência multiprofissional em saúde da família e comunidade. Rev Bras Ciênc Saúde [serial on the internet]. 2011 [cited 2018 Nov 5];15(3):32938. Available from: file:///D:/10834-17191-1-PB.pdf

15. Borges AMP, Salício VAMM, Gonçalves MANB, Lovato M. A contribuição do fisioterapeuta para - Programa de Saúde da Família - uma revisão da literatura. Uniciências. 2010;14(1):70-82.

16. Silva MAC, Chaves MA, Silva RSU. Grupo de gestante Pingo de Gente: uma experiência exitosa. South American Journal of Basic Education, Technical and Technological [serial on the internet]. 2018 [cited 2018 Nov 5];5(1):270-6. Available from: http://revistas.ufac.br/revista/index.php/SAJEBTT/ article/view/1658/1057

17. Toldrá RC, Cordone RG, Arruda, BA, Souto ACF. Promoção da saúde e da qualidade de vida com idosos por meio de práticas corporais. Mundo Saúde [serial on the internet]. 2014 [cited 2018 Nov 5];38(2):15968. Available from: http://bvsms.saude.gov.br/bvs/ artigos/mundo saude/promocao saude qualidade vida idosos.pdf

18. Carvalho Filha FSS, Nogueira LT, Viana LMM. HiperDia: adesão e percepção de usuários acompanhados pela Estratégia Saúde da Família. Rev RENE [serial on the internet]. 2011 [cited 2018 Nov 5];12(Spec):930-6. Available from: http://www. periodicos.ufc.br/rene/article/view/4380/3348

19. Souza CS, Stein AT, Bastos, GAN, Pellanda LC. Controle da pressão arterial em hipertensos do programa HiperDia: estudo de base territorial. Arq Bras Cardiol [serial on the internet]. 2014 [cited 2018 Nov 5];102(6):571-8. Available from: http:// www.scielo.br/pdf/abc/v102n6/pt 0066-782Xabc-102-06-0571.pdf
20. Rodrigues AD, Dallanora CR, Rosa J, Germani ARM. Sala de espera: um ambiente para efetivar a educação em saúde. Vivências: Revista Eletrônica de Extensão da URI [serial on the internet]. 2009 [cited 2018 Nov 5];5(7):101-6. Available from: http://www.reitoria.uri.br/ vivencias/Numero 007/ artigos/artigos vivencias 07/Artigo 13.pdf

21. Bezerra MIC, Lima MJMR, Lima YCP. A visita domiciliar como ferramenta de cuidado da fisioterapia na Estratégia Saúde da Família. Sanare (Sobral, Online) [serial on the internet]. 2015 [cited 2018 Nov 5];14(1):76-80. Available from: https://sanare. emnuvens.com.br/sanare/article/view/612/329

22. David MLO, Ribeiro MAG0, Zanolli ML, Mendes RT, Assumpção MS, Schivinsk CIS. Proposta de atuação da fisioterapia na saúde da criança e do adolescente: uma necessidade na atenção básica. Saúde Debate [serial on the internet]. 2013 [cited 2018 Nov 5];37(96):120-9. Available from: http://www.scielo. br/pdf/sdeb/v37n96/14.pdf

23. Marques KSF, Freitas PAC. A cinesioterapia como tratamento da incontinência urinária na unidade básica de saúde. Fisioter Mov [serial on the internet]. 2005 [cited 2018 Nov 5];18(4):63-7. Available from: https://periodicos.pucpr.br/index. $\mathrm{php/fisio/article/view/18646/18064}$

24. Gasparetto A, Pivetta HMF, Frigo LF, Braz MM, Padilha JF, Santos N, et al. Efeitos da fisioterapia com abordagem em grupo sobre a incontinência urinária feminina na atenção primária. Disciplinarum Scientia - Série: Ciências da Saúde [serial on the internet]. 2011 [cited 2018 Nov 5];12(1):59-70. Available from: https://www.periodicos.unifra.br/ index.php/disciplinarumS/article/viewFile/977/920

25. Teixeira RC, Corrêa, R0, Silva, EM. Percepções dos discentes de terapia ocupacional sobre a experiência de integração ensino-serviço-comunidade. Cad Bras Ter 0cup [serial on the internet]. 2018 [cited 2018 Nov 5];26(3):617-25. Available from: file:/// D:/1952-10657-1-PB.pdf
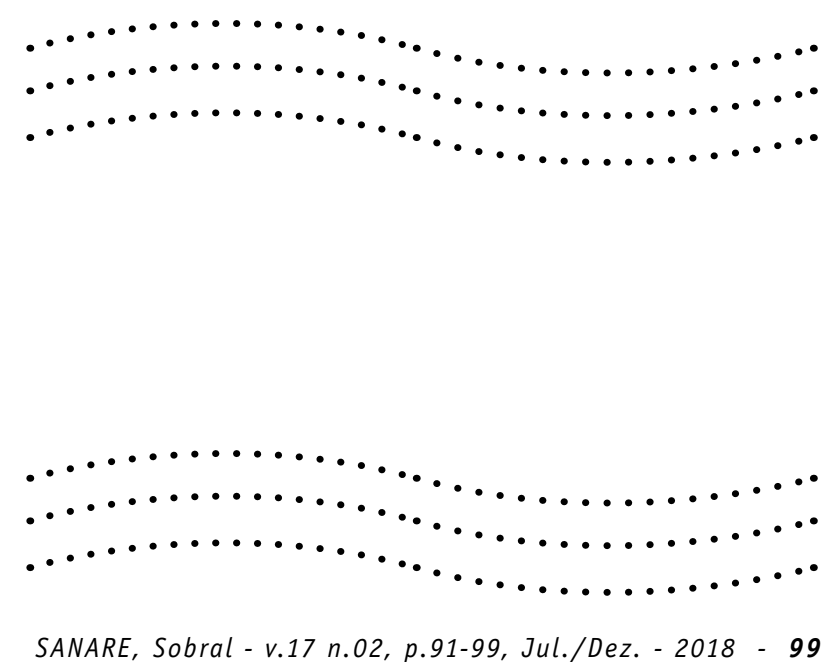